\title{
La evolución de la Sanidad Militar en Valencia durante La Guerra Civil Española (1936-1939)
}

\author{
García Ferrandis X. ${ }^{1}$, Munayco Sánchez AJ. ${ }^{2}$
}

Sanid. mil. 2011; 67 (4): 383-389; ISSN: 1887-8571

\begin{abstract}
RESUMEN:
Antecedentes y objetivos: Tras la insurrección militar del 18 de julio de 1936 el Ejército de la República quedó parcialmente desarticulado, resultando afectada la Sanidad Militar. En el caso de Valencia, ésta fue asumida rápidamente por el Comité Sanitario Popular, un organismo revolucionario que lideró la respuesta sanitaria de Valencia a la Guerra Civil. Posteriormente, el Gobierno Republicano procedió a recuperar el poder a través de medidas de carácter centralizador, como fue la militarización de algunos hospitales. Fue entonces cuando volvió a articularse una auténtica Sanidad Militar. El objetivo de este trabajo es estudiar la evolución de la Sanidad Militar en Valencia, una de las zonas republicanas más importantes. Material y métodos: Se analiza la documentación generada por los abundantes hospitales instalados en la ciudad de Valencia, estudiando los cambios que se produjeron tras la militarización de algunos centros sanitarios. Esta información ha sido consultada en el Archivo de la Excma. Diputación Provincial de Valencia. Resultados: La Sanidad Militar en Valencia durante la Guerra Civil española atravesó varias etapas, evolucionado desde la improvisación inicial a la posterior organización. Conclusiones: Se establece una relación bidireccional entre el grado de organización de la Sanidad Militar y la efectividad de la tropa.
\end{abstract}

PALABRAS CLAVE: Guerra Civil Española, Sanidad Militar, Milicianos, Militarización.

\section{The evolution of Military Health in Valencia during the Spanish Civil War (1936-1939)}

SUMMARY:

Precedents and purposes: After the military insurrection of July 18, 1936 the Republican Army remained partially dismantled, affecting Military Health. In case of Valencia, it was assumed rapidly by Sanitary Popular Committee, a revolutionary organism that led the sanitary response of Valencia to the Civil War. Later, the Republican Government proceeded to recover power throghout centralizing measures, as the militarization of some hospitals. At this the time an authentic Military Health returned to be articulated. The aim of this work is to study the evolution of Military Health in Valencia, one of the most important republican zones. Material and method: Documentation generated by the abundant hospitals installed in the city of Valencia is analyzed, studying as well changes that took place after militarization of some sanitary centers. This information has been consulted in the File of the County Council of Valencia. Results: Military Health in Valencia during the Spanish Civil War went through several stages, as it evolved from initial improvisation to later organization. Conclusions: A bidirectional relation is established between the degree of Military Health organization and troop's efficiency.

KEY WORDS: Spanish Civil War, Military Health, militiamen, militarization.

\section{INTRODUCCIÓN}

Con este trabajo pretendemos complementar desde el punto de vista de la Sanidad Militar la abundante bibliografía existente sobre la Guerra Civil Española (1936-1939). Hemos podido comprobar el tratamiento superficial de esta temática, tanto en obras de carácter general como en trabajos locales ${ }^{1-2}$.

Tan sólo en dos obras hemos encontrado referencias a la Sanidad Militar de ambos bandos durante la Guerra Civil ${ }^{3-4}$. En estos libros, sin embargo, se analiza la asistencia sanitaria en determinadas campañas, obviando el análisis de la evolución de la Sanidad Militar republicana.

\footnotetext{
${ }^{1}$ Doctor en Medicina y Cirugía. Universidad de Valencia. Valencia. España.

2 Cap. Médico. Unidad Médica Aérea de apoyo al despliegue-Madrid (UMAAD-MADRID). España.
}

Dirección para correspondencia: Xavier García Ferrandis. Correo electrónico: xagarfe@ alumni.uv.es

Recibido: 16 de febrero de 2011

Aceptado: 27 de junio de 2011
Hemos considerado, por tanto, que el análisis de la Sanidad Militar a lo largo de la contienda resulta muy interesante, especialmente en Valencia, una de las zonas de la retaguardia republicana más importante por el papel que asumió debido a esta condición geoestratégica y por su participación en las campañas para la toma de Teruel.

\section{LA EVOLUCIÓN DE LA GUERRA EN VALENCIA}

La insurrección militar del 18 de julio de 1936 produjo un vacío de poder y un clima de inestabilidad política en aquellas zonas donde fracasó. Ante la incapacidad del Gobierno republicano, los sindicatos y partidos del Frente Popular se encargaron de reconducir la situación política y armada para hacer frente a los militares sublevados.

Una consecuencia del alzamiento para el Ejército de la República fue la falta de oficiales, bien porque pasaron a formar parte del Ejército sublevado en aquellas zonas donde triunfó, bien por las purgas a que fueron sometidos en las zonas donde fracasó la insurrección ${ }^{5}$. El poder de los sindicatos y partidos durante el inicio de la guerra hizo que muchos de los nuevos dirigentes castrenses fueran elegidos entre sus afiliados y simpatizantes, sin que tuvieran conocimientos mili- 
tares. Además, los oficiales que habían mostrado muestras de legitimidad a la República quedaron integrados en las milicias populares, donde solo pudieron ejercer el mando parcialmente.

Resulta evidente, por tanto, la desarticulación que supuso la insurrección militar de julio de 1936 para el Ejército de la República, cuya Sanidad Militar también se vio afectada.

En Valencia, el 22 de julio de 1936 los partidos políticos integrantes del Frente Popular (Partido Comunista, Partido Socialista e Izquierda Republicana, entre otros) y los sindicatos UGT y CNT constituyeron el Comité Ejecutivo Popular, un organismo revolucionario que entre julio y diciembre de 1936 ejerció todas las competencias a través de varias delegaciones. En materia sanitaria, el Comité Ejecutivo creó la Delegación de Sanidad o Comité Sanitario Popular, que pasó a estar dirigida por Francisco Bosch Morata, líder del Partit Valencianista d'Esquerra y especialista en Análisis Clínicos. El secretario general era Rafael Vilar Fiol, odontólogo y otorrinolaringólogo y presidente del Sindicato Médico (UGT). Finalmente, Emilio Navarro Beltrán -médico del Hospital Provincial afiliado a la CNT-era el responsable de la gestión de los hospitales ${ }^{6}$.

De lo expuesto anteriormente se puede concluir que en Valencia la respuesta sanitaria más inmediata al estallido de la guerra fue articulada por tres médicos civiles, que gestionaron el Comité Sanitario Popular, un organismo revolucionario que gestionó la política sanitaria de Valencia durante los primeros meses de guerra.

Por otra parte, hay que señalar que la situación geoestratégica de Valencia durante la totalidad de la Guerra Civil condicionó el papel de la ciudad en la contienda. Así, Valencia fue una de las ciudades más importantes de la retaguardia republicana, lo que tuvo dos consecuencias. En primer lugar, una de las prioridades más inmediatas tras el estallido de la Guerra Civil fue organizar la respuesta sanitaria al nuevo contexto bélico, con el fin de aumentar la oferta sanitaria para poder atender la demanda asistencial por parte de los heridos y enfermos de guerra y de evacuados civiles. En este sentido, los partidos de izquierda y los sindicatos UGT y CNT habilitaron toda una red de instalaciones sanitarias, que fueron coordinadas y administradas por el Comité Sanitario Popular.

Además, la situación geoestratégica de la ciudad la convirtió desde el mismo verano de 1936 en un polo receptor de refugiados y evacuados de guerra -muchos de ellos enfermos o heridos- que huían de zonas de conflicto ${ }^{7}$.

En noviembre de 1936 el Gobierno de Largo Caballero se trasladó a Valencia, donde inició un progresivo proceso de centralización política que consistió básicamente en la asimilación de los comités revolucionarios mediante la creación de los consejos provinciales, unos organismos con las competencias recortadas ${ }^{8}$. En Valencia supuso la desaparición del Comité Sanitario Popular y la creación de la Consejería de Sanidad, integrada en el Consejo Provincial de Valencia. Este nuevo organismo en un primer momento estuvo dirigido por el médico cenetista Emilio Navarro Beltrán, aunque en octubre de 1937 fue sustituido por Manuel Alonso, en la órbita de Izquierda Republicana.

La Consejería de Sanidad, por tanto, pasó a gestionar desde principios de 1937 los hospitales distribuidos por la ciudad.

Otra medida centralizadora fue la militarización de las milicias, que fueron integradas junto con las Brigadas Internacionales en las denominadas Brigadas Mixtas, espina dorsal del Ejército Popular de la República. Era un ejército regular sometido a una férrea disciplina de guerra que sustituyó a las unidades militares espontáneas con poca disciplina y preparación. De hecho, el fracaso de las milicias valencianas en la toma de Teruel durante el invierno de 1936-1937 fue determinante en la decisión de Largo Caballero9.

Sin embargo, la medida de carácter centralizador que más nos interesa para el desarrollo de esta investigación fue la Orden del Ministerio de la Guerra de 23 de enero de 1937, que analizaremos posteriormente.

\section{LA SANIDAD MILITAR DESDE EL INICIO DE LA GUERRA HASTA 1937 EN MADRID}

La primera respuesta sanitaria a la guerra estuvo marcada por la inexperiencia y la improvisación ${ }^{10}$. Los expertos de la Sociedad de Naciones, en el informe que elaboraron sobre la situación sanitaria de la España republicana entre diciembre de 1936 y enero de 1937, definieron así esta primera etapa:

"Cuando ha estallado la sublevación, la República española se ha encontrado bruscamente privada de una parte importante de sus recursos médicos. Ha perdido de una manera casi completa su Servicio de sanidad Militar (...). Le han quedado aproximadamente, 10.000 médicos civiles, los cuales han realizado, en condiciones más difíciles, una obra de reorganización y de adaptación» ${ }^{11}$.

Es decir, durante las primeras semanas de conflicto la descomposición del Cuerpo de Sanidad Militar hizo que la sanidad civil se reorganizara con el objetivo de suplirlo sin dejar de lado los problemas sanitarios de la población civil. Los casos del farmacólogo Juan Planelles y del dermatólogo Julio Bejarano resultan muy representativos. El primero organizó la Sanidad Militar del Ejército Republicano del Centro cuando el Gobierno abandonó Madrid en noviembre de 1936. Por su parte, Bejarano, antiguo director general de Sanidad, llegó a ocupar la Comandancia de Sanidad del Ejército de la República, después de haber organizado la estructura sanitaria del Cuerpo de Carabineros ${ }^{12}$.

Esta adaptación de la sanidad civil no estuvo exenta, sin embargo, de algunos problemas derivados de la falta de experiencia en el ámbito militar, como por ejemplo el exceso de oferta sanitaria. En este sentido, la misión internacional se pronunció en los siguientes términos:

«Se ha desarrollado mucho la creación de nuevas instalaciones sanitarias utilizando conventos o edificios privados requisados. Se reconoce hoy que se ha multiplicado demasiado el número de pequeños hospitales. El proyecto es reducir su número, a fin de que el esfuerzo sanitario se ejerza de una manera más eficaz. Cualquiera que sea el conjunto de los hospitales, reúne para los heridos un total de 25.000 camas. Un gran número vacantes» ${ }^{13}$.

En este mismo sentido apuntó José Estellés Salarich, médico valenciano que ocupaba el cargo de secretario general técnico de la Dirección General de Sanidad cuando estalló la guerra:

«Fundaban hospitales los partidos politicos, las centrales sindicales y todas las organizaciones en las que había alguien con fantasia, generosidad (...). De estas instituciones, unas llegaron a funcionar bien, otras no eran más que el pretextos 
para que persones generosas y bien intencionadas, pero deficientemente orientadas, se entretuvieran dedicando inocentemente sus esfuerzos a los auxilios de guerra. Al cabo de unos dias habia muchos hospitales (...) demasiados hospitales» ${ }^{14}$.

Otro testimonio importante en esta dirección fue el de Guillermo Luna. Luna ocupó la Jefatura de Sanidad de Madrid cuando el Gobierno abandonó la capital. En noviembre de 1938, con ocasión del segundo aniversario de aquellos hechos, se publicó una entrevista en la revista militar La Voz de la Sanidad del Ejército de Maniobra en la que Luna describía la situación sanitaria con la que se había enfrentado desde finales de julio de 1936 y que tuvo que reconducir:

«Puede decirse que no existía un barrio en Madrid que no tuviese sus hospitales (...). Todo este esfuerzo desorganizado no servía, a pesar del gran entusiasmo de todos, para tener bien atendidas las necesidades sanitarias de las milicias,(...) mientras habia hospitales donde la alimentación y cuidados eran excelentes, otros carecían de lo más preciso» ${ }^{15}$.

Podemos resumir la situación descrita anteriormente como la existencia de un exceso de oferta sanitaria con los peligros que eso conllevaba, es decir, la dispersión de material y personal médico con pérdida de eficacia sanitaria como resultado final.

El Gobierno tomó conciencia de esta situación sanitaria anómala desde bien pronto, y el 22 de agosto de 1936 emitió un decreto que facultaba al Ministerio de Trabajo, Sanidad y Previsión a frenar la excesiva proliferación de hospitales en Madrid ${ }^{16}$. En el artículo primero de esta disposición se enumeraban los 14 centros sanitarios a lo que se les reservaba el nombre de «hospitales de sangre», donde se tenían que evacuar exclusivamente los heridos de guerra. El artículo tercero establecía una lista de nueve «hospitales de convalecencia», donde tenían que recibir atención los heridos leves y los enfermos de guerra. Estos centros no podían denominarse «hospitales de sangre» y tenían que disponer de un mínimo de 50 ingresados para no ser clausurados. Finalmente, el Decreto anunciaba el cierre de todos los establecimientos no incluidos en el mismo y la prohibición expresa de habilitar hospitales nuevos.

De todo lo expuesto anteriormente podemos concluir que el Gobierno a lo largo del mismo verano de 1936 inició un proceso de centralización sanitaria.

Guillermo Luna desde la Jefatura de Sanidad de Madrid procedía a cumplir el Decreto del Gobierno:

«En Madrid habia una infinidad de hospitales que, por su escasa capacidad y falta de medios (...) no reunian las condiciones necesarias para un buen tratamiento de nuestros heridos; además, mantenían un exceso de personal en sus plantillas (...). Había que cerrarlos, y con sus camas y efectos aumentar la capacidad de los que quedaban» ${ }^{17}$.

Uno de estos grandes hospitales fue instalado en el hotel Ritz. Dirigido por Estellés Salarich, atendió en un primer momento a los heridos de los diferentes frentes. Asimismo, sirvió para descongestionar los hospitales General y Clínico. Otros centros sanitarios de gran capacidad fueron el del Colegio de San Fernando (situado en la carretera de Colmenar) y el Hospital del Rey, especializado en enfermedades infecciosas ${ }^{18}$. Finalmente, cabe destacar el Hospital de Chamartín, donde en abril de 1937 el médico argentino Gregorio Bermann habilitó una pequeña clínica para neurosis de guerra que rápidamente reunió a un 70 enfermos de los frentes del Centro ${ }^{19}$.

\section{LA SANIDAD MILITAR DESDE EL INICIO DE LA GUERRA HASTA 1937 EN VALENCIA}

La situación sanitaria en Valencia fue muy parecida al estallar la guerra, ya que también allí proliferaron notablemente las instalaciones médicas por iniciativa de los partidos políticos, sindicatos y comités obreros. Buen ejemplo de esta realidad es la red de postas de urgencia que se tejió en Valencia desde finales de julio de 1936. Estaba integrada por 26 unidades instaladas en toda suerte de edificios, tanto civiles como religiosos (la plaza de toros, el Monte de Piedad, cines, el colegio de los Salesianos, etc.). Asimismo, en Valencia y alrededores se habilitaron hasta nueve hospitales de sangre: el Hospital «Blanquer», el Hospital «Pasionaria», el Hospital de Sangre de Izquierda Republicana, el Hospital de Sangre de la Cruz Roja y los hospitales de sangre de Benimaclet, de Benimàmet, de Natzaret, de Mislata y de Burjassot ${ }^{20}$. Como se ha señalado anteriormente, esta intensa reorganización de las infraestructuras sanitarias respondió a un aumento de la demanda asistencial debido a los numeroso heridos y enfermos de guerra que llegaron a Valencia.

Podemos concluir, por tanto, que durante la etapa «revolucionaria» de la guerra (julio-diciembre de 1936) proliferaron abundantes hospitales en la ciudad de Valencia, por lo que el exceso de oferta sanitaria también fue un problema en esta zona republicana durante los primeros meses de conflicto.

En la figura 1 se puede apreciar la distribución de los hospitales de sangre en Valencia a finales del año 1936.

Sin embargo, la solución a este problema sanitario en el caso de Valencia fue radicalmente diferente al de Madrid. El Ministerio de Trabajo, Sanidad y Previsión no tenia competencias en la ciudad, ya que el control político correspondía al Comité Ejecutivo Popular, lo que hizo que no se pudiera aplicar el Decreto sobre el control de la proliferación de hospitales. Además, la autoridad sanitaria competente -el Comité Sanitario Popular- iba en la dirección opuesta, dado que aceptaba sistemáticamente todos los ofrecimientos de cualquier organización de la ciudad y la provincia a habilitar nuevos hospitales, comprometiéndose a abastecerlos de material, con la dispersión de recursos que suponía ${ }^{21}$.

Como hemos indicado anteriormente, uno de los primeros cometidos del Gobierno cuando se trasladó a Valencia fue «enfriar» la revolución y tomar el control de la situación con el objetivo de ganar la guerra. En materia sanitaria, esta centralización pasaba por frenar la proliferación de centros sanitarios, para lo que el Gobierno ordenó que no se instalaran hospitales con menos de 100 camas «por no poder hacer frente a los gastos que ocasionan los que tienen menor número» ${ }^{22}$. Sin embargo, la realidad fue que los hospitales con poca capacidad continuaron funcionando ${ }^{23}$, lo que obligó al Gobierno a desarrollar una poderosa herramienta legislativa: la Orden del Ministerio de la Guerra de 23 de enero de $1937^{24}$. Mediante esta disposición se procedía a la incautación de todos los hospitales de sangre con una capacidad superior a las 300 camas, que pasaban a estar bajo control militar bajo el nombre de «Hospitales de Sanidad Militar.» Asimismo, se disponía el cierre de todos los centros sanitarios que no tuvieran un mínimo de 300 plazas. 


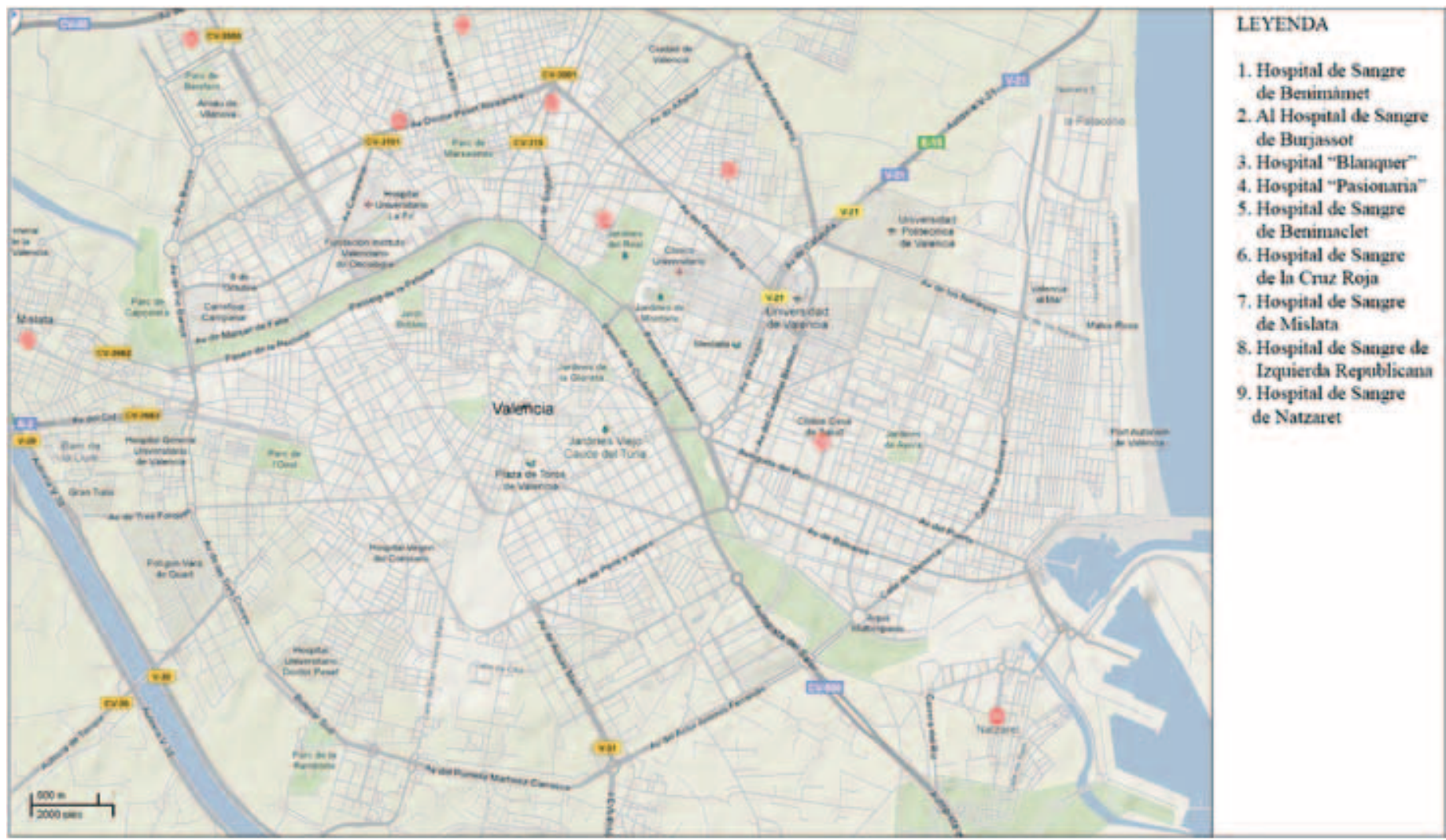

Figura 1. Hospitales de sangre en Valencia durante la Guerra Civil Española.

Del análisis de esta Orden se desprende la intención del Gobierno de centralizar los grandes hospitales existentes en la zona republicana a través de un proceso de militarización. Sin embargo, si comparamos esta disposición con la que se aplicó en Madrid durante el verano de 1936 nos daremos cuenta de que la centralización sanitaria que se llevó a cabo a partir de 1937 fue mucho más intensa, dado que el Decreto de 1936 ordenaba el cierre de los hospitales con una capacidad inferior a las 50 camas, mientras que la Orden de 1937 establecía el límite de viabilidad en 300.

Con el objeto comprobar el efecto que tuvo la Orden del Ministerio de la Guerra de 23 de enero de 1937 en los hospitales de sangre valencianos, hemos esquematizado el número de camas de los mismos en la tabla 1 .

Así pues, la Orden suponía el cierre del 67\% de los hospitales de sangre de la ciudad de Valencia, una cifra elevada pero mucho inferior al número de centros sanitarios clausurados en la provincia ${ }^{25}$.

Tabla 1. Capacidad de los hospitales de sangre de la ciudad de Valencia.

\begin{tabular}{|lc|}
\hline \multicolumn{1}{|c|}{ Hospital de Sangre } & N. $^{\circ}$ Camas \\
\hline Izquierda Republicana & 300 \\
Benimàmet & 30 \\
Natzaret & 30 \\
«Pasionaria» & 300 \\
Cruz Roja & 20 \\
«Blanquer» & 400 \\
Mislata & 90 \\
Burjassot & 100 \\
Cruz Roja & 20 \\
\hline
\end{tabular}

Sin embargo, la mayoría de los hospitales de sangre valencianos desaparecieron a lo largo de 1937 por motivos económi$\cos \sin$ que hiciera falta aplicar la Orden. En este sentido hay que recordar que los centros sanitarios recibían una subvención del Ministerio de la Guerra (para cubrir los gastos de heridos y enfermos de guerra) y del Ministerio de Sanidad (para atender a evacuados heridos o enfermos). Esta cantidad se otorgaba por paciente ingresado, de manera que los hospitales con una elevada ocupación eran los que más recibían más financiación. La dificultad para el mantenimiento económico de las instalaciones por falta de pacientes ingresados fue el verdadero motivo que obligó a cerrar a muchos centros sanitarios valencianos, dado que, cuando las autoridades sanitarias militares centralizaron a los enfermos y heridos militares en los Hospitales de Sanidad Militar, la ocupación de los hospitales de sangre descendió drásticamente y los hizo económicamente inviables.

En la ciudad de Valencia tenemos un claro ejemplo en el Hospital de Sangre de Benimàmet: «En fecha de hoy, y según lo acordado ha sido cerrado este Hospital de Sangre por su pequeña cabida y solo contar con 13 hospitalizados, los cuales pasan al Hospital de Sanidad Militar de Mislata ${ }^{26}$.

Si nos fijamos en la tabla 1 comprobaremos como el Hospital de Mislata contaba con 90 camas y, sin embargo, no fue clausurado tal y como establecía la Orden, sino que se utilizó para derivar pacientes de hospitales cercanos que sí fueron cerrados.

En general, podemos afirmar que el cierre de los hospitales de sangre valencianos supuso un claro proceso de centralización de recursos sanitarios por motivos económicos.

Por el contrario, los hospitales de sangre valencianos con una capacidad superior a las 300 camas sí que resultaron afectados por 
la disposición del Ministerio de la Guerra y pasaron a convertirse en hospitales militares. El Hospital «Pasionaria» es un buen ejemplo en la ciudad de Valencia. Fundado por el PCE en noviembre de 1936 como hospital de sangre, a principios de 1937 fue transformado en el Hospital Militar número 2. El Hospital de Sangre de Izquierda Republicana pasó igualmente a estar bajo control de la Sanidad Militar. El caso del Hospital «Blanquer» difiere sustancialmente, ya que este centro, de forma excepcional, había estado gestionado por la Sanidad Militar desde finales de 1936. La militarización de 1937, no obstante, lo transformó en el Hospital Militar número 3.

De lo expuesto anteriormente se puede concluir que durante 1937 tuvo lugar la reorganización de la Sanidad Militar valenciana que, en la práctica, supuso la militarización de gran parte de la sanidad civil.

Una vez consolidada la Sanidad Militar, los hospitales militares valencianos quedaron bajo mando de la Jefatura de los Servicios Sanitarios Médicos de la Tercera División Orgánica del Ejército de la República (Figura 2).

\section{LA SANIDAD MILITAR VALENCIANA EN 1938}

Si durante 1936 la Sanidad Militar valenciana se había caracterizado por la desorganización y 1937 había sido el año de la reorganización y consolidación, podemos afirmar que durante 1938 tuvo lugar la extensión y ampliación de la misma.

A lo largo de 1938 la Sanidad Militar tejió una auténtica red de hospitales militares por toda la región de Levante, siete de los cuales estaban ubicados en la ciudad de Valencia y alrededores ${ }^{(1)}$. Estos centros también recibían el nombre de «Clínica Militar» y eran identificados con números (Hospital Militar número 1, Hospital Militar número 2, etc.).

En la tabla 2 podemos apreciar algunos de los hospitales militares de Valencia y su distribución.

Todos los hospitales militares estaban coordinados por el Hospital Militar de la ciudad, que era el de mayor capacidad e importancia, por lo que recibía el nombre de «Hospital Militar número 1». Por su parte, el Hospital Provincial de Valencia también fue afectado por la militarización de 1937 y fue considerado desde entonces por las autoridades militares como una clínica militar más ${ }^{(2)}$.

La Sanidad Militar valenciana durante 1938 estuvo muy influenciada por la intensidad de las campañas republicanas. La ofensiva sobre Teruel durante el invierno de 1937-1938, la pérdida posterior

(1) Sabemos el Hospital Militar número 7 estaba situado en Villena, Alicante (A.D.P.V., D.6.1 c. 14). La extensa documentación consultada, sin embargo, nos ha impedido situar la totalidad de clínicas militares habilitadas. Por este motivo, el autor principal en el curso de su investigación doctoral recurrió a testimonios orales, entrevistando a dos testigos de aquella época. Uno de ellos, capitán médico, participó en la defensa de Madrid bajo las órdenes de Estellés Salarich y posteriormente cubrió la retirada del Ejército de Maniobra durante la campaña del Maestrazgo. El otro testigo entrevistado era trabajador de las atarazanas del puerto de Valencia, objetivo militar de la aviación alemana. Desgraciadamente ninguno de los dos pudo aportar luz a la distribución los hospitales militares en Valencia.

(2) Para conocer el funcionamiento del Hospital Militar y del Hospital Provincial de Valencia durante la Guerra Civil española consúltese: García Ferrandis X. La reorganització de l'assistència medicoquirúrgica de la ciutat de València durant la Guerra Civil espanyola (1936-1939). Valencia: Universidad de Valencia. Tesis doctoral, 2010: 193-240 y 261-266.

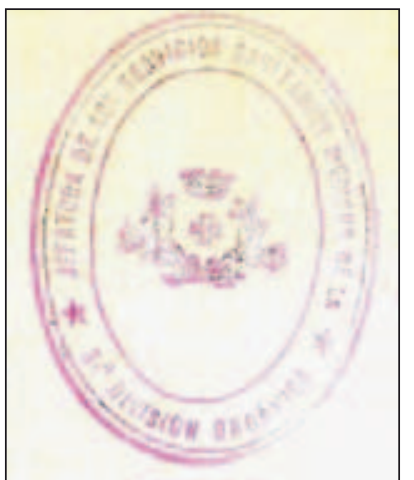

Figura 2. Sello de la $3^{a}$ División (Fuente: Archivo de la Excma. Diputación Provincial de Valencia, I-2.4 caja 8, legajo 32).

Tabla 2. Red de hospitales militares en Valencia en 1938

\begin{tabular}{|lc|}
\hline \multicolumn{1}{|c|}{ Centro Sanitario } & Hospital Militar \\
\hline Hospital Militar & 1 \\
Hospital Militar «Pasionaria» & 2 \\
Hospital Militar «Blanquer» & 3 \\
Hospital de Sanidad Militar de Godella & 4 \\
Hospital Militar de Benimaclet & 15 \\
Facultad de Medicina & Hospital Militar Base \\
Hospital Provincial & Clínica Militar del Hospital \\
& Provincial \\
\hline
\end{tabular}

Fuente: García Ferrandis, 2010, p. 262.

de la ciudad y el repliegue de las tropas bajo condiciones meteorológicas durísimas con temperaturas de hasta 18 grados bajo cero ${ }^{27}$ colapsaron el Hospital Militar de Valencia. A esta saturación contribuyó una epidemia de fiebre tifoidea que se declaró en Valencia el verano de $1938^{28}$.

En la figura 3 se puede observar una ficha de un soldado que participó en la toma de Teruel. Nótese como el diagnóstico («pie de trinchera») está en consonancia con lo que acabamos de exponer.

La saturación del Hospital Militar hizo que la Facultad de Medicina se habilitara como Hospital Militar Base, donde fueron evacuados numerosos participantes en la batalla de Teruel.

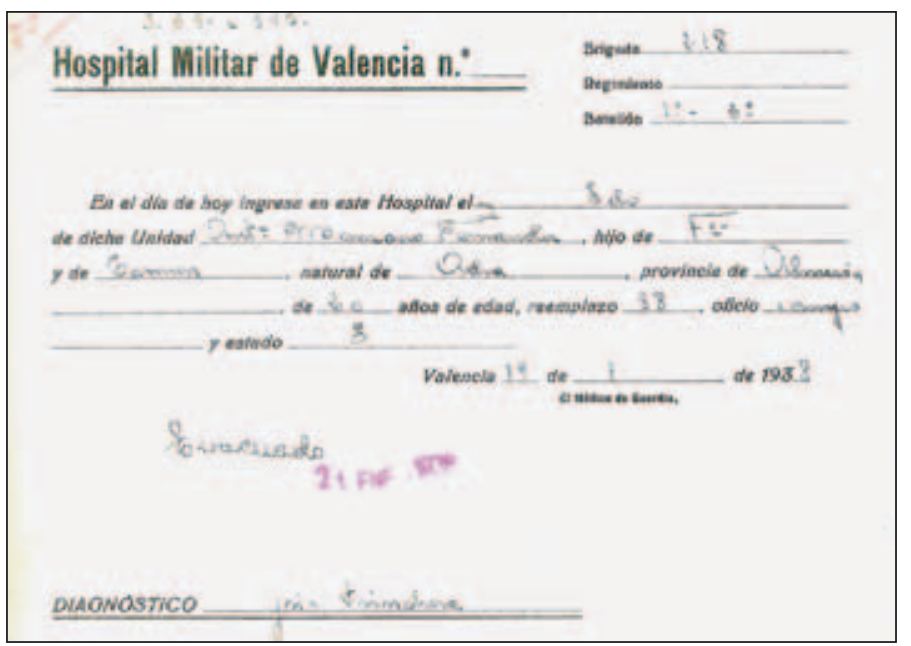

Figura 3. Ingreso en el Hospital Militar de Valencia (Fuente: Archivo de la Excma. Diputación Provincial de Valencia, I-2.4 caja 5, legajo 19). 


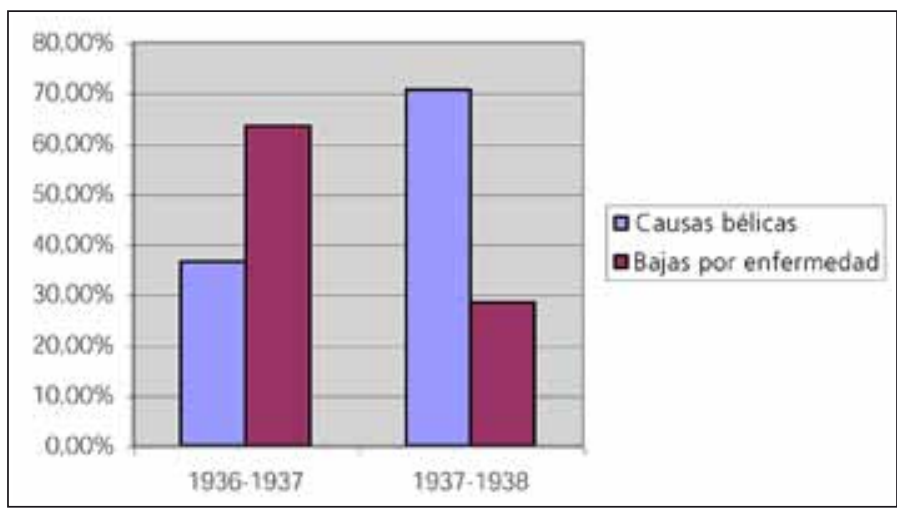

Figura 4. Causas de baja en las dos campañas republicanas para tomar Teruel (Fuente: elaboración propia a partir de datos consultados en A.D.P.V., D.6.1 caja 13 y I-2.4 caja 5, legajo 19).

Después de la pérdida de Teruel, el Estado Mayor franquista inició una ofensiva para llegar al Mediterráneo, partiendo así el territorio de la República. Esta ofensiva hizo replegarse al Ejército de Maniobra de la República en dirección sudeste por toda la provincia de Castellón en la denominada campaña del Maestrazgo. Durante esta campaña el Ejército de Maniobra absorbió al Ejército de Levante, aunque una vez estabilizado el frente en julio de 1938 prevaleció el último nombre. Para recibir a los heridos evacuados en los combates de la campaña del Maestrazgo, la Sanidad Militar valenciana habilitó el Hospital de Evacuación del Ejército de Levante, que tenía dos sedes: el Hospital Militar Base y el Hospital Militar número 2 (antiguo Hospital «Pasionaria»).

Para terminar, en la figura 4 presentamos una comparación de las causas de baja en las dos campañas republicanas que se llevaron a cabo durante la Guerra Civil con el fin de tomar Teruel.

Como se puede observar, en la segunda campaña sobre Teruel aumentaron notablemente las bajas por causas bélicas y disminuyeron los casos de soldados afectados por enfermedades comunes, pese a que el invierno de 1937-1938 fue mucho más riguroso que el anterior.

En la tabla 3 se recogen los cuadros clínicos más frecuentes. Hay que tener en cuenta que los datos referidos a la primera campaña pertenecen al período de tiempo comprendido entre agosto de 1936 y febrero de 1937 (siete meses), mientras que la información existente de la segunda campaña tan solo abarca los meses de enero y mayo de 1938. En el gráfico consultado en Sanidad Militar las heridas por metralla fueron consignadas como heridas por arma de fuego (h.p.a.f.). Aquí hemos mantenido esa clasificación al objeto de poder comparar.

\section{RESULTADOS}

La Sanidad Militar en Valencia atravesó por cuatro etapas a lo largo de la Guerra Civil: improvisación, consolidación, extensión y reorganización.

Tabla 3. Cuadros clínicos más frecuentes en las campañas para tomar Teruel.

\begin{tabular}{|lcc|}
\hline & h.p.a.f. / metralla & Infecciones respiratorias \\
\hline Primera campaña & 431 & 445 \\
Segunda campaña & 503 & 224 \\
\hline
\end{tabular}

Fuente: elaboración propia a partir de datos consultados en Sanidad Militar 2010; 66 (4), 248 y en A.D.P.V., caja 5, legajos 19 y 20.
La etapa de improvisación (julio-diciembre de 1936) abarca la respuesta sanitaria más temprana a la Guerra Civil, que se basó en la reorganización de la sanidad civil para cubrir la ausencia de Sanidad Militar. Sin embargo, este iniciativa civil supuso un exceso de oferta sanitaria con la consiguiente dispersión de recursos médicos y una pérdida de eficacia como resultado final.

Aunque la etapa de consolidación empezó progresivamente durante la capitalidad valenciana, no puede hablarse de una auténtica Sanidad Militar hasta enero de 1937 cuando, en un contexto de fuerte centralización política, se militarizaron los grandes hospitales de sangre valencianos.

La etapa de extensión de la Sanidad Militar tuvo lugar aproximadamente entre enero y julio de 1938, cuando en la ciudad de Valencia y alrededores existía una extensa red de hospitales militares.

A partir de julio de 1938, podemos hablar de una etapa de reorganización de la Sanidad Militar en Valencia. Las causas hay que buscarlas en la saturación del Hospital Militar número 1 debido a la caída de Teruel y al repliegue de las tropas republicanas.

Por otra parte, conviene resaltar que la militarización de las milicias para aumentar su rendimiento militar fue efectiva. Solo así se explica el mayor aumento de las bajas por causas bélicas durante la segunda campaña para tomar Teruel, ya que los soldados, mejor preparados y más disciplinados, entraron en combate con más frecuencia. A esta mayor efectividad militar contribuyó la organización de la Sanidad Militar, que se tradujo en una menor incidencia de enfermedades en la tropa. Además, la mayor eficacia del Ejército Republicano repercutió directamente en la Sanidad Militar, ya que cambió el patrón de paciente atendido por la misma, pasando de un combatiente enfermo a uno herido por arma de fuego.

\section{CONCLUSIONES}

De todo lo expuesto anteriormente se puede concluir que:

1. La centralización sanitaria impulsada por el Gobierno de la República en enero de 1937 tuvo un claro precedente en Madrid durante el otoño de 1936. Los buenos resultados de esta gestión sanitaria centralizada animó a las autoridades a extender el control de los recursos sanitarios de una forma mucho más intensa por todo el territorio de la República. Tras un primer intento fallido, la centralización de los hospitales civiles valencianos solo consiguió ser efectiva tras la militarización de los de mayor capacidad. Sin embargo, la baja capacidad de la mayoría de los centros sanitarios de Valencia hizo que las autoridades militares fueran permisivas en la aplicación de la legislación referente al cierre de hospitales.

2. La eficaz articulación de la Sanidad Militar republicana se manifestó durante la toma de Teruel en enero de 1938; a su vez, la efectividad militar influyó en el Cuerpo de Sanidad. Queda establecida, por tanto, una relación bidireccional entre la organización de la Sanidad Militar y la efectividad de la tropa.

\section{FUENTES HISTÓRICAS Y BIBLIOGRAFÍA}

1. Preston P. La Guerra Civil española. Barcelona: Ediciones Debate, 2006: 287-290.

2. Girona A, Santacreu JM (coords). La Guerra Civil en la Comunidad Valenciana. Valencia: Editorial Prensa Valenciana, 2006. 
3. Rojo V. Algunos aspectos de la Sanidad Militar durante las operaciones de Teruel En: Laboratorios Beecham (coord). Los médicos y la medicina en la Guerra Civil española. Monografías Beecham. Madrid: Ediciones Saned, 1986: 141-146.

4. Massons JM. Historia de la Sanidad Militar española. Barcelona: Ediciones Pomares Corredor, 1994: 393-394.

5. Alpert M. El ejército republicano en la guerra civil. Barcelona: Ibérica de Ediciones y Publicaciones, 1977: 329.

6. García Ferrandis X. La reorganització de l'assistència medicoquirúrgica de la ciutat de València durant la Guerra Civil espanyola (1936-1939). Valencia: Universidad de Valencia. Tesis doctoral, 2010: 55-56.

7. Ibidem, 63.

8. Decreto de 23 de diciembre de 1936 sobre la creación de los consejos provinciales, Gaceta de la República de 25 de diciembre de 1936 (núm. 360), p. 1102 y ss.

9. García Ferrandis X, Munayco AJ. La asistencia sanitaria en el frente de Terue durante la primera campaña republicana (agosto 1936-febrero 1937). Sanidad Militar 2010; 66 (4): 245-249.

10. Barona JL, Bernabeu J. La salud y el Estado. El movimiento sanitario internacional y la administración española (1851-1945). Valencia: Universidad de Valencia 2008: 257.

11. Rapport sur la mission sanitaire en Espagna (1937). Consultado en Barona JL, Bernabeu J. Ciencia y sanidad en la Valencia capital de la República. Valencia: Universidad de Valencia, 2007: 101.

12. Estellés Salarich J. La Sanidad del Ejército republicano del Centro. En: Laboratorios Beecham (coord). Los médicos y la medicina en la Guerra Civil española. Monografías Beecham. Madrid: Ediciones Saned, 1986: 44.

13. Rapport sur la mission sanitaire en Espagna (1937). Consultado en Barona JL, Bernabeu J. Ciencia y sanidad en la Valencia capital de la República. Valencia: Universidad de Valencia, 2007: 107.

14. Estellés Salarich J. La Sanidad del Ejército republicano del Centro. En: Laboratorios Beecham (coord). Los médicos y la medicina en la Guerra Civil española. Monografías Beecham. Madrid: Ediciones Saned, 1986: 41.

15. 7 de noviembre (una charla con el camarada Luna) (1938). Consultado en Barona JL, Bernabeu J. Ciencia y sanidad en la Valencia capital de la República. Valencia: Universidad de Valencia, 2007: 157.
16. Decreto de 22 de agosto de 1936 sobre el control de la proliferación de hospitales en Madrid, Gaceta de Madrid de 23 de agosto de 1936 (núm. 236), pp. 1430 y 1431.

17. 7 de noviembre (una charla con el camarada Luna) (1938). Consultado en Barona JL, Bernabeu J. Ciencia y sanidad en la Valencia capital de la República. Valencia: Universidad de Valencia, 2007: 160.

18. Estellés Salarich J. La Sanidad del Ejército republicano del Centro. En: Laboratorios Beecham (coord). Los médicos y la medicina en la Guerra Civil española. Monografías Beecham. Madrid: Ediciones Saned, 1986: 47-50.

19. Rodríguez Lafora G. La psiquiatría y neurología de guerra y de la revolución. Sus problemas y soluciones. Revista de Sanidad de Guerra 1937; 4, 127.

20. García Ferrandis X. La reorganització de l'assistència medicoquirúrgica de la ciutat de València durant la Guerra Civil espanyola (1936-1939). Valencia: Universidad de Valencia. Tesis doctoral, 2010: 73-79, 137-144.

21. El CSP rechaza la centralización sanitaria propuesta por el Comité de la localidad de Navajas (Valencia). 1936. Archivo de la Diputación Provincial de Valencia (A.D.P.V); D.6.1. caja 24, Hospitales de Sangre.

22. El CSP rechaza la instalación de hospitales con capacidad inferior a 100 camas según la Orden Circular de 24 de noviembre de 1936 sobre hospitales. Diario Oficial del Ministerio de la Guerra, núm. 247, p. 369. A.D.P.V; D.6.1. caja 24, Hospitales de Sangre.

23. El director del Hospital de Sangre de Alberique (Valencia) comunica el ingreso de milicianos. 1937. A.D.P.V; D.6.1. caja 24, Hospitales de Sangre.

24. Orden del Ministerio de la Guerra sobre la militarización de hospitales civiles, Gaceta de la República de 26 de enero de 1937 (núm. 26), p. 510.

25. García Ferrandis X. La asistencia sanitaria en la provincia de Valencia durante la Guerra Civil española (1936-1939). Llull 2011; 34 (73): 13-38.

26. Cierre del hospital de sangre de Benimàmet. 1937. A.D.P.V; D.6.1. caja 33, Hospitales de Sangre.

27. Massons JM. Historia de la Sanidad Militar española. Barcelona: Ediciones Pomares Corredor, 1994: 394.

28. García Ferrandis X. La reorganització de l'assistència medicoquirúrgica de la ciutat de València durant la Guerra Civil espanyola (1936-1939). Valencia: Universidad de Valencia. Tesis doctoral, 2010: 268. 